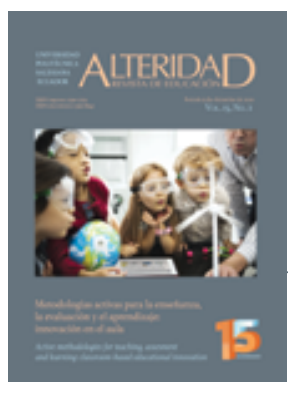

\title{
Twitter como recurso metodológico en Educación Superior: Una experiencia educativa con estudiantes de Trabajo Social
}

\section{Twitter as a methodological resource in Higher Education: An educational experience with Social Work students}

Dr. José Alberto Gallardo-López es docente e investigador de la Universidad Pablo de Olavide (España) (jagallop@upo.es) (https://orcid.org/0000-0003-3243-1676)

(D) Dr. Fernando López-Noguero es docente e investigador de la Universidad Pablo de Olavide (España) (flopnog@upo.es) (https://orcid.org/0000-0002-1124-8613)

Recibido: 2020-01-31 / Revisado: 2020-06-03 / Aceptado: 2020-06-08 / Publicado: 2020-07-01

\section{Resumen}

El perfil del estudiante universitario está experimentando cambios significativos que obligan a las universidades a replantearse nuevas estrategias metodológicas para favorecer los procesos de enseñanza-aprendizaje. En este trabajo se analizan los principales resultados derivados de un proyecto de innovación apoyado en la incorporación de Twitter como recurso metodológico colaborativo, colectivo e innovador en Educación Superior. El objetivo general del estudio es explorar el impacto educativo del uso de Twitter como recurso didáctico, y cómo perciben los estudiantes la utilidad de este instrumento integrado en una metodología participativa. La muestra se compone de 137 estudiantes de primer curso del Grado en Trabajo Social de la Universidad Pablo de Olavide, de Sevilla (España). Se trata de un estudio ex post facto y transversal, que parte de una metodología descriptiva, utilizando instrumentos de recogida y análisis de la información de corte cualitativo y cuantitativo. En líneas generales, los resultados obtenidos tras el análisis de la participación, de los comentarios elaborados por los estudiantes y del contenido audiovisual compartido, abordan cuestiones centradas en la influencia del uso de Twitter para la mejora de la dinámica natural del desarrollo académico en la asignatura de Introducción a la Pedagogía Social, observando como aporta numerosos beneficios en la creación colectiva del aprendizaje, desde la colaboración, la participación y la cohesión de grupo.

Descriptores: Twitter, Educación Superior, redes sociales, aprendizaje colaborativo, metodología participativa.

\section{Abstract}

The profile of the university student is undergoing significant changes that are forcing universities to rethink new methodological strategies to favour teaching-learning processes. In this paper we analyze the main results derived from an innovation project supported by the incorporation of Twitter as a collaborative, collective and innovative methodological resource in Higher Education. The general objective of the study is to explore the educational impact of the use of Twitter as a teaching resource, and how students perceive the usefulness of this instrument integrated into a participatory methodology. The sample is composed of 137 firstyear students of the Degree in Social Work of Pablo de Olavide University, Seville (Spain). It is an ex post facto and transversal study, which starts from a descriptive methodology, using instruments for the collection and analysis of qualitative and quantitative information. In general terms, the results obtained after the analysis of participation, of the comments made by the students and of the shared audiovisual content, address questions centred on the influence of the use of Twitter for the improvement of the natural dynamics of academic development in the subject of Introduction to Social Pedagogy, observing how it provides numerous benefits in the collective creation of learning, from collaboration, participation and group cohesion.

Keywords: Twitter, Higher Education, innovative methodologies, social networks, collaborative learning, participatory methodology.

Forma sugerida de citar: Gallardo-López, J.A., \& López-Noguero, F. (2020). Twitter como recurso metodológico en Educación Superior: Una experiencia educativa con estudiantes de Trabajo Social. Alteridad, 15(2), 174-189. https:// doi.org/10.17163/alt.v15n2.2020.03 


\section{Introducción y estado de la cuestión}

\subsection{Perfil del estudiante universita- rio en la era tecnológica}

En la actualidad, la sociedad no podría entenderse sin su vinculación con los últimos desarrollos tecnológicos y sus aplicaciones, y se caracteriza por tener ciudadanos interconectados de manera continua, gracias, entre otras cuestiones, al fácil acceso a internet y a la comunicación de telefonía móvil. Esta nueva forma de comunicación $\mathrm{y}$ de entender las relaciones ha transformado de manera considerable el modo de concebir lo que nos rodea y de adaptarnos a las nuevas realidades (Halliwell, 2020). De acuerdo con Martínez y Acosta (2011) y López-Noguero y Cobos (2016), las Tecnologías de las Información y de la Comunicación (TIC) posibilitan acceder de forma instantánea a una gran cantidad de información, que debe ser gestionada teniendo en cuenta que el exponencial crecimiento de estas nuevas tecnologías está reconfigurando las situaciones comunicativas y los procesos de enseñanza y aprendizaje.

Vivimos en una sociedad eminentemente digitalizada $y$, por tanto, debemos ser conscientes de que aspectos relativos a la convivencia en comunidad, como son los valores, las emociones, las relaciones que establecemos, pero también la manera de comunicarnos y el tratamiento que le demos a la información que creamos y compartimos, se ven totalmente influenciados por la forma en la que utilizamos las herramientas tecnológicas que nos permiten el acceso a estos contextos digitales. Lo queramos o no, las nuevas tecnologías son medios que educan y socializan (Morón et al., 2017), por lo que es indispensable trabajar en competencias relacionadas con estas cuestiones desde la Enseñanza Superior.

En la actualidad, los estudiantes que cursan programas de estudios universitarios forman la primera generación educada en convivencia natural con los nuevos avances tecnológicos. Esta cir- cunstancia obliga a las universidades a adaptarse a las nuevas particularidades de una sociedad cambiante y en continua evolución, transformándose y desarrollando su praxis educativa en estas nuevas realidades (González-Hernando et al., 2020).

Por todo ello, las metodologías deben contextualizarse teniendo en cuenta las necesidades de este nuevo perfil de estudiante, considerando todas las características de esta nueva generación. En este sentido, este novedoso contexto educativo requiere de pedagogías más participativas en todas las etapas educativas, incluyendo la Enseñanza Superior. Pedagogías que giren en torno al aprendizaje social, que generen espacios para la enseñanza, el aprendizaje y la evaluación de competencias, con un carácter activo y motivador (Noguera, 2015; Paredes et al., 2020).

De acuerdo con Martínez-Rodrigo y RayaGonzález (2013), el nuevo perfil de estudiante universitario requiere enseñanzas que permitan conseguir competencias como la capacidad de trabajar en equipos interdisciplinares, crear y compartir información y contenidos educativos en diferentes formatos interactivos y dinámicos, ser partícipes activos y protagonistas de su propio aprendizaje.

Estudios recientes de carácter internacional demuestran el interés que suscita este nuevo perfil del estudiante universitario. Las investigaciones de alto impacto científico, que han sido publicadas en los últimos años, analizan cuestiones como el uso de los smartphones en contextos universitarios por parte de los estudiantes, con el objetivo de fomentar el uso de tecnologías móviles en las actividades docentes en Educación Superior (Aguirre et al., 2019). Por otro lado, Jami y Taheri (2019), desarrollan un estudio en el que miden los efectos de los rasgos de personalidad de los estudiantes universitarios en el comportamiento a la hora de intercambiar conocimientos en las redes sociales, mientras Gavilán et al. (2017) analizan cómo las redes sociales pueden convertirse en un instrumento muy Útil para empoderar a la ciudadanía, desde una perspectiva activa y crítica. 
Como podemos comprobar, las nuevas tendencias en investigación consideran los emergentes perfiles de comportamiento de los alumnos y sus nuevas realidades sociales, tratando de aproximarse a resultados empíricos que demuestren la necesidad de adaptación por parte de las instituciones educativas de Enseñanza Superior. Sobre esta cuestión, autores como Valeria et al. (2018) llevan a cabo un estudio longitudinal centrado en los factores cognitivos y motivacionales que influyen en el riesgo de abandono en estudiantes universitarios, tratando de identificar qué estrategias cognitivas pueden reforzarse para favorecer el aprendizaje y minimizar el abandono de los estudios.

\subsection{Metodologías para nuevas reali- dades educativas en las aulas universitarias}

Transcurridos ya casi veinte años desde la Declaración de Bolonia, que inició la creación de un nuevo Espacio Europeo de Educación Superior (EEES), se puede apreciar cómo la función docente ha experimentado cambios importantes relacionados con la adopción de metodologías innovadoras donde el principal protagonista del proceso educativo pasa a ser el propio estudiante. Entendemos por metodologías innovadoras, aquellas que se alejan de la visión tradicional de la enseñanza y del aprendizaje, y proponen nuevas formas de abordar la docencia, valiéndose de todo tipo de recursos para mejorar los procesos educativos.

En este sentido, el nuevo perfil del docente de Educación Superior debe dotar a sus estudiantes de ciertas aptitudes que trasciendan el mero conocimiento científico-académico, así como promover el papel activo de las personas ante su propio aprendizaje (García \& Carmona, 2014; Garcias et al., 2020).

Al respecto, García y García (2015) reflexionan acerca de los principios pedagógicos innovadores que se dan en Educación Superior, y ponen el foco de atención a la necesaria tran- sición hacia concebir una educación universitaria centrada en el estudiante y en sus procesos formativos. Por todo lo expuesto, las TIC y las posibilidades educativas que genera el acceso a internet, son elementos clave que inciden directamente en las dinámicas educativas actuales, mejorando la calidad de los procesos pedagógicos y funcionando como catalizador de aprendizajes activos y colaborativos (Alonso \& Alonso, 2014; Juma et al., 2019; Cotán et al., 2020).

Por otro lado, es preciso fomentar técnicas de intercambio de conocimientos, experiencias y sentimientos en la resolución de problemas de forma colaborativa en el aula, a través de una metodología participativa, propiciando la implicación activa de los estudiantes durante el desarrollo de la enseñanza y el aprendizaje, impulsando la actitud crítica ante la información y promoviendo la adquisición de una serie de competencias específicas relacionadas con lo digital.

En este sentido, autores como Bautista y Cipagauta (2019) inciden en la importancia de la innovación docente y en la capacidad que deben tener para generar nuevos entornos de aprendizaje que favorezcan la autogestión del conocimiento por parte de los estudiantes. Estos investigadores identifican el aprendizaje colaborativo y el aprendizaje basado en proyectos como las metodologías didácticas innovadoras más utilizadas en Educación Superior, y advierten de una tendencia hacia el uso de metodologías de aprendizaje activo en las prácticas pedagógicas.

En opinión de Rodríguez y Restrepo (2015), la presencia de las TIC como recurso al servicio de la educación en la universidad ha experimentado un aumento a lo largo de los últimos años, principalmente por las numerosas ventajas que ofrece al desarrollo de los procesos de enseñanza, aprendizaje y evaluación de conocimientos y competencias. Entre los principales beneficios que aportan las TIC en estos contextos educativos podemos señalar que éstos facilitan el acceso a los contenidos didácticos, y aumentan la cantidad y variedad de recursos educativos en distintos formatos audiovisuales (Castro \& 
Chirino, 2011; Sánchez-Saus \& Crespo, 2018; Arango et al., 2020).

De igual forma, los docentes y los estudiantes utilizan los recursos tecnológicos disponibles para establecer comunicaciones que mejoran la planificación y el desarrollo de la formación, y estimulan la construcción crítica del pensamiento y la autonomía desde un enfoque de educación continua (Bidarian et al., 2011; Corujo-Vélez et al., 2020).

Por otro lado, existen estudios sobre el uso de las TIC en la enseñanza que alertan de la importancia de educar en el correcto empleo de las nuevas tecnologías, ya que existen una serie de riesgos asumidos como puede ser el uso excesivo y descontrolado que genera problemas sociales y educativos, relacionados principalmente con comportamientos adictivos, dependencia, inseguridad y mala gestión de la información accesible (Garrote et al., 2018; Plaza de la Hoz, 2018; Gairín \& Mercader, 2018), así como los problemas derivados de la manipulación y el control de las redes digitales (Machado, 2018; Da Silveira, 2017).

\subsection{Las redes sociales como recurso metodológico: El caso de Twitter}

Hoy en día, las redes sociales son utilizadas de forma habitual para la interacción social en espacios socioeducativos, destacando entre la población universitaria la red social Twitter. La incorporación de esta red social en Educación Superior ha sido objeto de muchos estudios en la literatura especializada, entre los que destacan los de Grosseck y Holotescu (2008), que señalan el potencial educativo que esta red social proporciona al favorecer el desarrollo de las habilidades de síntesis de la información en un contexto de interacción inmediata.

Otros autores como Thoms y Eryilmaz (2015) o Htay et al. (2020), indican que este tipo de herramientas fomenta el aprendizaje autónomo de los estudiantes, convirtiéndolos en auténticos protagonistas del hecho educati- vo, potenciando el aprendizaje informal (Tess, 2013; Dommett, 2019), facilitando las interacciones sociales de todos los implicados desde el trabajo colaborativo (García-Suárez et al., 2015; Fernández-Ferrer \& Cano, 2019; Malik et al., 2019) y fomentando la integración de nuevas metodologías en Educación Superior.

Estudios científicos actuales de alto impacto, recogen evidencias de la utilidad de Twitter en espacios de Educación Superior. Por ejemplo, podemos señalar a Eaton y Pasquini (2020), que indagan sobre cómo las comunidades educativas fomentan el aprendizaje y el desarrollo académico de los estudiantes en un entorno participativo, donde la estructura del liderazgo es compartida por la naturaleza bidireccional de las relaciones online.

Por otro lado, Abella-García et al. (2019) ahondan su investigación en cómo la red social Twitter puede aumentar potencialmente la reflexión, el aprendizaje y la colaboración entre estudiantes universitarios, contribuyendo a mejorar la calidad de los procesos de enseñanzaaprendizaje. Por otra parte, Hortigüela-Alcalá et al. (2019), indagan sobre el uso pedagógico de las redes sociales y cómo Twitter influye significativamente en el aumento de la motivación, el aprendizaje, la participación y el grado de logro académico de los estudiantes. En esta línea de investigación, Gleason y Manca (2019) abordan la cuestión del uso educativo de Twitter y su repercusión hacia el cumplimiento de objetivos educativos como la alfabetización digital y el desarrollo profesional desde un paradigma participativo.

Finalmente, es interesante resaltar el trabajo de Acar et al. (2019), donde exploran la relación entre el uso de las redes sociales y la creatividad, y obtienen datos significativos que concluyen que un perfil activo en la red social Twitter, donde el estudiante expresa sus ideas y opiniones, reflexiona y crea un ambiente discursivo sobre temáticas educativas de interés, está relacionado con una actividad creativa de alto nivel.

De acuerdo con Vázquez-Cano y Sevillano (2019), la interacción social de los estudiantes en la universidad, y los procesos de ense- 
ñanza-aprendizaje que se dan en este entorno de Educación Superior, trasciende los espacios físicos hacia nuevos contextos virtuales complementarios, basados en la ubicuidad y facilitados por las nuevas tecnologías emergentes.

El perfil actual de los estudiantes se caracteriza por acceder e interactuar, de igual forma, en entornos físicos y virtuales. Además, es muy frecuente que el usuario de internet tenga un perfil activo en redes sociales ya que ayuda a mantener contactos profesionales o de relación e intercomunicación personal de una manera participativa y proactiva. Además, en estos espacios es posible crear y compartir información y conocimientos valiosos, entendiendo que todo aprendizaje significativo surge de la interacción con el medio social y su relación con el contexto educativo de los estudiantes (Van der Krogt, 1998; O'Keeffe, 2019; Al-Dheleai et al., 2020). Por ello, es necesario innovar en metodologías docentes que se valgan de este tipo de herramientas de creación de contenido y divulgación de la información (Adams et al., 2018).

Las redes sociales digitales facilitan la interacción y el aprendizaje continuo, y fomentan la comunicación online (Siemens \& Weller, 2011; Ruiz \& Fachinetti, 2018), creando comunidades de aprendizaje donde los participantes generan contenido, interactúan entre ellos y comparten el conocimiento (Van Pujienbroek et al., 2014; Cela-Ranilla et al., 2017). En este sentido, Fernández-Ferrer y Cano (2019) indican que la utilidad de las redes sociales en procesos formativos de Educación Superior está justificada, debido a su gran potencial como herramienta didáctica al servicio de estudiantes y docentes, desde una perspectiva participativa y colaborativa.

Este tipo de redes, utilizadas como herramienta para la Educación Superior, proporcionan un valor añadido a la enseñanza ya que implican atracción social y motivación para los estudiantes, fomentan la comunicación y el establecimiento de grupos de trabajo, posibilitando acciones comunes a nivel docente (De Haro, 2009; Saeed \& Sinnappan, 2011; García \& García, 2012; Domingo-Coscollola et al., 2020).
Concretamente, Twitter es considerada una herramienta de micro-blogging, que posibilita escribir y consultar mensajes de manera pública y gratuita, conocidos como tweets. El tamaño de los mensajes está restringido a 280 caracteres, pero la red social Twitter permite también incluir elementos multimedia como imágenes, pequeños videos o enlaces a páginas web. Autores como Freites (2017) consideran que "Twitter no es sólo una red social, sino un medio masivo de interacción" (p. 70) ya que, sobre la estructura de intercambio de información de dicha plataforma, encontramos el uso de los denominados hashtags o etiquetas, además de otras opciones para el usuario como la indicación de menciones a perfiles de usuario de docentes o la posibilidad de seguimiento de otros perfiles de interés.

Los estudios de Pérez et al. (2012) describen las ventajas del uso de esta red social para la comunidad universitaria, entre las que se encuentran el aumento de la atención en las actividades académicas, un incremento importante en el sentido de compromiso y pertenencia al grupo clase, y el fomento de la participación activa en la construcción del conocimiento. Asimismo, el uso de esta red social permite expandir los procesos de enseñanza-aprendizaje más allá de un espacio físico y de un horario determinado (Peña et al., 2018; Ribeiro et al., 2020).

De igual forma, es sustancial señalar que la información que se crea en esta red social puede ser comentada por cualquier usuario, pudiendo generarse hilos argumentales de comentarios. Así puede compartirse fácilmente mediante la opción retweet, expandiendo de manera exponencial el impacto de los tweets que la comunidad genera. En definitiva, la accesibilidad y el sencillo manejo de esta herramienta de red social digital, facilita su integración como parte metodológica en la docencia universitaria, promoviendo pedagogías participativas con una clara orientación hacia el impulso del aprendizaje y la construcción colectiva del conocimiento (Prestridge, 2014; Vizcaíno-Laorga et al., 2019; Rojprasert et al., 2020). 
Finalmente, si abordamos el uso de las redes sociales en el ámbito educativo, no podemos obviar que también existen aspectos negativos que debemos tener en cuenta al diseñar propuestas metodológicas. Al respecto, Marín-Díaz y Cabero-Almenara (2019) reflexionan sobre la necesidad de generar más investigación científica que aporte modelos de referencia para la utilización de estas tecnologías en ámbitos universitarios. Otro aspecto fundamental a tener en cuenta es que, de inicio, existe una visión negativa generalizada por parte del profesorado y del alumnado para incorporar las redes sociales online como elemento metodológico en enseñanzas de Educación Superior (Andreev et al., 2020; Van Dijck, 2020), unido a una preocupación del usuario sobre cuestiones relacionadas con la seguridad, la privacidad o la falta de control sobre los mensajes emitidos en la red (Sai et al., 2020; Miller, 2020; Ozer et al., 2020).

\section{Material y métodos}

El objetivo general de esta investigación es examinar el impacto educativo del uso de Twitter como recurso didáctico en Educación Superior, y cómo perciben los estudiantes la utilidad de esta tecnología inserta en una metodología participativa.

La muestra que compone este estudio está formada por un total de 137 estudiantes de primer curso del Grado en Trabajo Social de la Universidad Pablo de Olavide, de Sevilla (España). Concretamente, 67 estudiantes del grupo o línea de docencia 1 (L1) y 70 estudiantes de grupo o línea de docencia 2 (L2). En este sentido, indicar que, según los datos recogidos en la Memoria Académica 2018/2019 de la Universidad Pablo de Olavide (2019), hay un total de 705 estudiantes matriculados en el Grado en Trabajo Social, repartidos en los cuatro cursos de este Grado. La muestra seleccionada representa un $18,2 \%$ del total de la población matriculada en estos estudios superiores.

La investigación se enmarca en un proyecto de innovación docente denominado
«Twitter como herramienta didáctica innovadora y de construcción colectiva del conocimiento en Educación Superior», llevado a cabo en la Universidad Pablo de Olavide durante el curso académico 2019/2020. El proyecto consta de una serie de acciones o actividades didácticas relacionadas con Twitter, que se realizaron durante el progreso de la asignatura Introducción a la Pedagogía Social.

Las actividades fueron realizadas de forma grupal, a fin de favorecer el aprendizaje colaborativo y la cooperación de la comunidad educativa. Para ello, los docentes fomentaron, en diversos momentos de las sesiones, la interacción de los estudiantes a través de la red social Twitter. En este sentido, durante el desarrollo de las sesiones de la asignatura, los estudiantes escribieron tweets relativos a los contenidos tratados en la sesión utilizando hashtags (\#) para identificar mensajes sobre un tema específico. Los hashtags que se establecieron como protocolo metodológico de actuación fueron (\#IPS) (\#L1) (\#L2), todos ellos junto a la mención @Lopez_Noguero.

En diversos momentos de las sesiones, los estudiantes sintetizaron ideas, ampliaron la información disponible y trabajaron preconceptos de forma cooperativa, ya que en muchas ocasiones redactaron grupalmente los tweets y «retuitearon», y comentaron o marcaron como «me gusta» las publicaciones de los compañeros.

Con estas actuaciones, se trató de expandir los procesos educativos desarrollados más allá del espacio y el tiempo del aula universitaria, promoviendo que los estudiantes ingresen e interactúen en un universo más amplio, el de la web 2.0 (López-Noguero \& Cruz-Díaz, 2018). Al final de algunas sesiones, los docentes propiciaron una puesta en común vía Twitter, a modo de resumen, de los contenidos más importantes publicados. Esta tarea facilitó la labor de síntesis, generando una bitácora de lo expuesto que, en muchas ocasiones, es de gran utilidad para los estudiantes.

De igual forma, los docentes utilizaron la cuenta de Twitter para publicitar y anunciar cuestiones relativas a la asignatura (recordar tra- 
bajos o actividades pendientes, compartir la guía docente, facilitar alguna información sobre la asignatura, etc.). Además, se favoreció la formulación de preguntas, difusión de enlaces de interés y recomendaciones de cuentas de expertos en temas concretos, todo ello con el objetivo de dinamizar y mediar en el aprendizaje de forma didáctica.

La metodología de investigación utilizada para recopilar y analizar los datos generados en esta experiencia educativa es descriptiva, utilizando instrumentos de recogida y análisis de la información de corte cualitativo y cuantitativo. En este sentido, por un lado, se llevó a cabo un análisis descriptivo detallado de la actividad de los estudiantes en esta experiencia, considerando cuestiones como la cantidad de tweets y hashtags generados por la comunidad educativa implicada, la vinculación del contenido con la temática de la asignatura, exposición de argumentos y aportaciones informativas consideradas de interés para el estudio. Para este cometido se utilizó la herramienta Twitter Analitycs para valorar los datos relativos a la actividad de los estudiantes implicados durante la experiencia docente. Por otro lado, se diseñó un cuestionario de satisfacción para la recogida de datos, que fue sometido a un proceso de validación mediante juicio de expertos y aplicado a la muestra a través del aula virtual de la universidad utilizando la aplicación Google Forms. Igualmente, el cuestionario fue sometido a la prueba Alfa de Cronbach para medir su fiabilidad, obteniendo una puntuación de 0.887 que la posiciona como aceptable (Mateo, 2004).

Para el tratamiento estadístico de los datos recogidos en esta encuesta de satisfacción se utilizó el software Statistical Package for the Social Sciences (SPSS V26), llevando a cabo análisis descriptivos básicos (porcentajes de respuesta, media, mediana, moda, desviación y varianza) que, en líneas generales, reflejan la importancia que otorgan los estudiantes a este tipo de experiencias.

Además, bajo un enfoque cualitativo, se analizó la información de los comentarios de los estudiantes sobre el uso de Twitter en la asignatura de Introducción a la Pedagogía Social. Para ello, y tratando de aproximarnos a los planteamientos de Bardin (2002), se creó un sistema de codificación en función de unas categorías de análisis relacionadas con los contenidos clave de la asignatura impartida: Planteamientos teóricos de interés, referencias a autores fundamentales de la pedagogía social, metodologías para la intervención socioeducativa, aprendizajes destacados, menciones entre compañeros/as, dudas planteadas, propuestas y sugerencias.

\section{Análisis y resultados}

A continuación, se presentan los datos recopilados en la investigación realizada con el objetivo conocer el impacto educativo que ha tenido el uso de Twitter en la asignatura de Introducción a la Pedagogía Social del Grado en Trabajo Social.

\subsection{Análisis descriptivo de la activi- dad de los estudiantes en Twitter}

En primer lugar, podemos comprobar que el perfil del docente responsable del proyecto de innovación experimentó un significativo aumento en la cantidad de tweets, impresiones de tweets, visitas al perfil, menciones y nuevos seguidores, desde que se inició la experiencia educativa en el aula en octubre de 2019 y finalizó en enero de 2020.

Estos datos demuestran el aumento de la participación de los estudiantes en esta red social durante el desarrollo de la asignatura, creando y compartiendo una gran cantidad de contenido. Como ejemplo de ello, si atendemos a los 100 últimos tweets de los estudiantes categorizados en los diferentes hashtags que utilizaron para identificar los comentarios (\#IPS, \#L1 y \#L2), podemos comprobar cómo un total de 82 aportaciones son en formato de texto y utilizando la combinación \#IPS \#L1, estas aportaciones recibieron 17 comentarios y 1 retweet. Por otro lado, teniendo en cuenta la combinación \#IPS \#L2, de los últimos 100 tweets escritos, 52 son en formato de texto y acumulan un total de 6 comentarios y 37 retweets. 


\section{Gráfico 1. Evolución del perfil del docente @Lopez_Noguero}

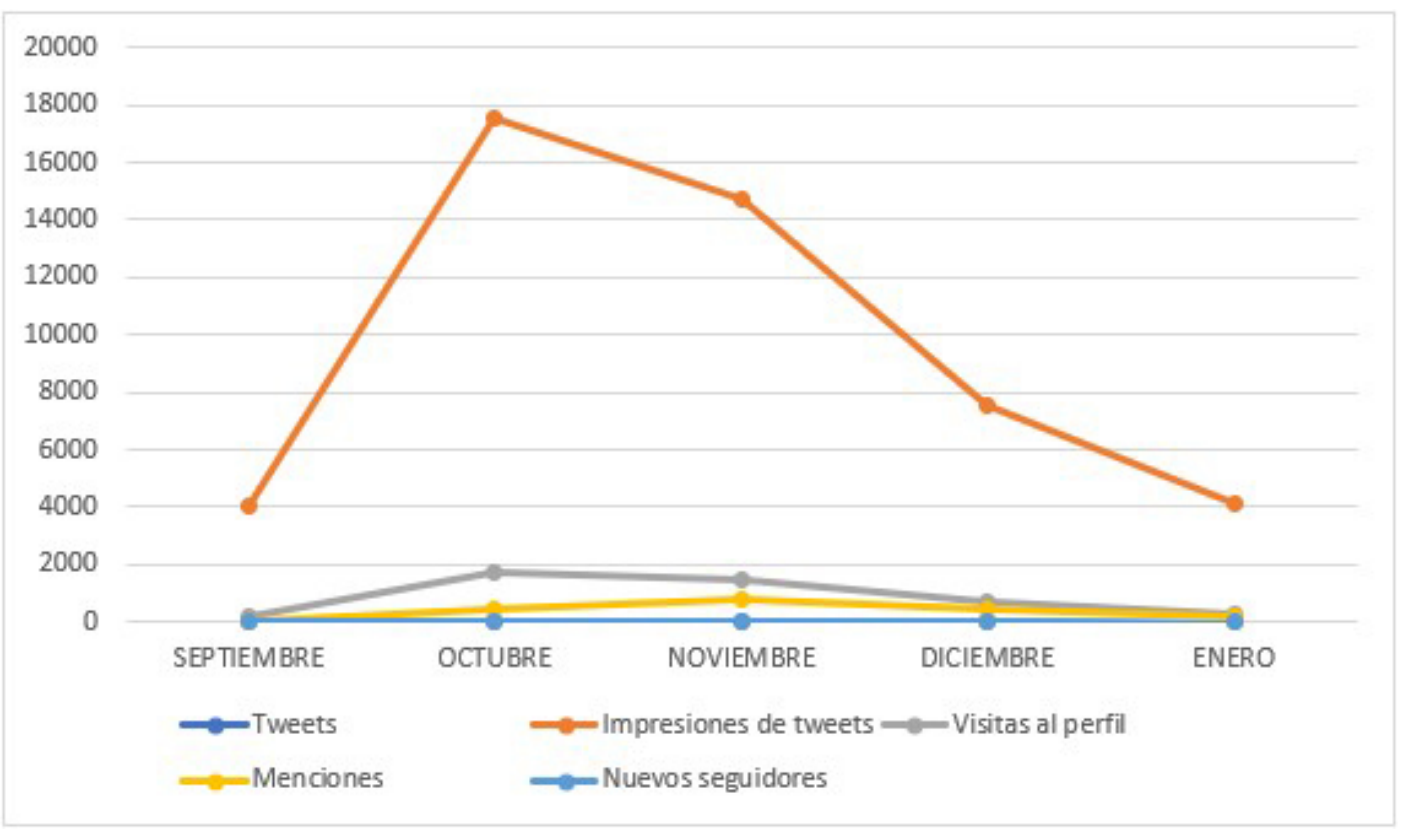

Fuente: Elaboración propia a partir de Twitter Analytics.

A continuación, podemos observar una recopilación de algunos de los tweets creados por los estudiantes:

La pedagogía tiene un importante valor en la educación que a día de hoy no se ha terminado tanto como para integrarla de manera formal en el ámbito educativo. (E1)

La pedagogía social es una ciencia teórica y práctica. (E2)

Otra de las funciones del trabajador social es prevenir las posibles causas de conflicto, tanto individual como colectivo. (E3)

Esta asignatura me parece muy interesante $\mathrm{e}$ importante. Creo que a través de ella se conocen los pilares fundamentales para el cambio de la sociedad y cómo saber intervenir con ésta. (E4)

Gracias a la dinámica grupal que hemos realizado en clase he conocido mejor a mis compañeros y se ha creado un vínculo muy bonito. (E5)
El análisis descriptivo de la información que proporcionan los tweets de los estudiantes manifiesta la difusión de conocimiento y de aspectos relevantes relacionados con la pedagogía social, y refleja el fomento de la comunidad de aprendizaje y de la cohesión grupal. Al finalizar la experiencia, los estudiantes realizaron una evaluación del proceso a través de la respuesta a una pregunta abierta sobre la utilidad de la herramienta Twitter. Las respuestas emitidas por la muestra participante encuentran la experiencia, en líneas generales, como positiva y altamente gratificante, como confirma esta selección de opiniones de los estudiantes:

Creo que Twitter ha sido una gran herramienta en la asignatura, me ha permitido conocer los distintos puntos de vista de mis compañeros y así conocer mejor la Pedagogía Social. (E6)

Desde mi punto de vista, la red social Twitter ha sido de gran ayuda para la asignatura de Introducción a la Pedagogía Social, ya que, al terminar las clases escribíamos dos o tres 
tweets que resumieran en pocas palabras lo que habíamos aprendido ese día. De esta forma, conseguíamos que nos quedará mucho más clara la materia dada en ese día y así salíamos de clase con las ideas claras. (E7)

Después de poner los tweets, nos tocaba interaccionar con los compañeros. Para mí este es uno de los momentos más útiles y divertidos de la actividad, ya que podíamos comentar que nos parecen las opiniones del resto, ver quiénes teníamos en común la misma opinión, quiénes no... (E8)

En mi opinión, la utilización de Twitter como una herramienta educativa es algo innovador que nunca había realizado anteriormente, pero me ha parecido una aportación muy positiva para la enseñanza de la asignatura, ya que nos ha servido para interaccionar entre los compañeros, además de aprender de una forma más colaborativa, dinámica e interactiva. (E9)

La utilización de la herramienta Twitter me ha parecido verdaderamente novedosa, dinámica y amena. Creo que es útil porque nos hace repasar el temario aprendido de una manera divertida. (E10)

Analizando las aportaciones, apreciamos cómo los alumnos valoran positivamente la integración de esta herramienta en la dinámica participativa de la asignatura. Particularmente destacan la posibilidad que les ha ofrecido para conectar e interactuar con otros estudiantes y conocer sus opiniones respecto a los contenidos tratados en clase, aprecian el aprendizaje colaborativo que se ha generado, el dinamismo y la interactividad, y manifiestan un interés significativo ante su uso didáctico, destacando los aspectos lúdicos y creativos que proporcionan las redes sociales en estos contextos universitarios.

\subsection{Análisis de los datos del cues- tionario de satisfacción sobre el uso de Twitter en la universidad}

Al finalizar la asignatura, los estudiantes realizaron una encuesta de satisfacción relativa al uso de la herramienta Twitter como instrumento didáctico, en la que participaron un total de 107 estudiantes, 16 hombres y 91 mujeres. Los 8 ítems que componen el cuestionario recogen información sobre distintas dimensiones de estudio como son: El conocimiento previo de la herramienta Twitter por parte de los estudiantes, la facilidad de uso durante la experiencia educativa, la conveniencia del protocolo establecido para organizar las aportaciones y la utilidad de la red social como parte integrada en la metodología docente, entre otras. Las respuestas a los diferentes ítems están en formato tipo Likert, con valoraciones ordinales que van de 1 a 4 , siendo 1 «completamente en desacuerdo» y 4 «completamente de acuerdo».

Ante la pregunta de si conocían con anterioridad la red social Twitter, un $80,4 \%$ de los encuestados responden estar completamente de acuerdo con la afirmación propuesta para este ítem. Por lo que entendemos que la gran mayoría de los encuestados ya partían con un conocimiento previo de la herramienta, y sabían utilizarla con fluidez, aunque un significativo $8,4 \%$ desconocía completamente esta aplicación.

Por otra parte, los datos recopilados confirman que al $86,9 \%$ de la muestra les resultó fácil utilizar Twitter durante el desarrollo de la asignatura (ítem 2). El 13,1\% restante, expresa haber tenido dificultades a la hora de utilizar la red social.

Este último dato acerca del ítem 2, anima a reflexionar sobre las posibles causas que han podido obstaculizar la dinámica planteada. En este sentido, los resultados obtenidos indican que los protocolos establecidos para establecer las comunicaciones en la red social Twitter fueron adecuados (ítem 3). Ya que un 49,5\% está completamente de acuerdo con la afirmación de este ítem, y un $46,7 \%$ de acuerdo.

En lo referente a los ítems 4. «Utilizar la herramienta Twitter como parte de la metodología de la asignatura me ha parecido útil» y 5. «Gracias a esta actividad con Twitter, hemos podido afianzar y ampliar los conocimientos 
sobre el contenido de la asignatura», podemos decir que los resultados son bastante positivos. Aunque hay un porcentaje significativo de estudiantes que no han considerado útil las actividades planteadas utilizando esta red social $(25,23 \%)$, o que indican que no les ha supuesto una mejora en afianzar y ampliar los conocimientos sobre la asignatura $(31,8 \%)$. Estos datos obligan a replantear el diseño de esta actividad en el marco de la asignatura para futuros cursos académicos, tratando de solventar las dificultades surgidas y mejorando el sistema de enseñanzaaprendizaje planteado. Es fundamental, motivar a todos y a cada uno de los estudiantes para que se puedan implicar en procesos colaborativos de una manera útil para su aprendizaje.

Tabla 1. Frecuencias y porcentajes parta el ítem 1 «Ya conocía la red social Twitter como usuario/a antes de usarla en clase de Pedagogía Social»

\begin{tabular}{|c|c|c|c|c|}
\hline & & Frecuencia & Porcentaje & $\begin{array}{l}\text { Porcentaje } \\
\text { acumulado }\end{array}$ \\
\hline \multirow{5}{*}{ Válido } & Completamente en desacuerdo & 9 & 8,4 & 8,4 \\
\hline & En desacuerdo & 2 & 1,9 & 10,3 \\
\hline & De acuerdo & 10 & 9,3 & 19,6 \\
\hline & Completamente de acuerdo & 86 & 80,4 & 100 \\
\hline & Total & 107 & 100 & \\
\hline
\end{tabular}

Fuente: Elaboración propia a partir de los datos de la encuesta de satisfacción.

Gráfico 2. Porcentajes de respuesta para el ítem 4. «Utilizar la herramienta Twitter como parte de la metodología de la asignatura me ha parecido útil»
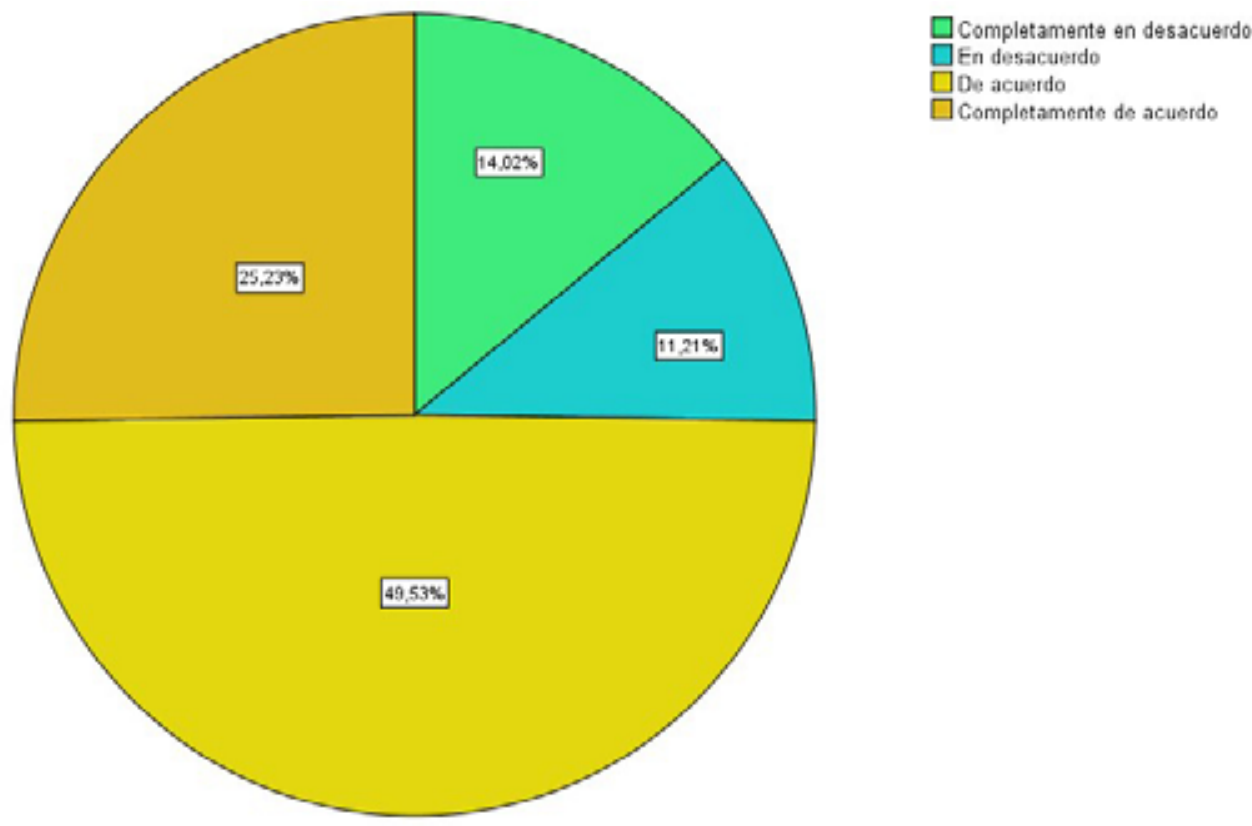

$\square \mathrm{En}$

$\square$ Completamente de acuerdo

Fuente: Elaboración propia a partir de los datos de la encuesta de satisfacción. 
Tabla 2. Frecuencias y porcentajes parta el ítem 5. "Gracias a esta actividad con Twitter, hemos podido afianzar y ampliar los conocimientos sobre el contenido de la asignatura»

\begin{tabular}{|c|c|c|c|c|}
\hline & & Frecuencia & Porcentaje & $\begin{array}{l}\text { Porcentaje } \\
\text { acumulado }\end{array}$ \\
\hline \multirow{5}{*}{ Válido } & Completamente en desacuerdo & 15 & 14 & 14 \\
\hline & En desacuerdo & 19 & 17,8 & 31,8 \\
\hline & De acuerdo & 50 & 46,7 & 78,5 \\
\hline & Completamente de acuerdo & 23 & 21,5 & 100 \\
\hline & Total & 107 & 100 & \\
\hline
\end{tabular}

\section{Fuente: Elaboración propia a partir de los datos de la encuesta de satisfacción.}

Respecto al ítem 6. «La herramienta Twitter nos ha servido para interaccionar entre nosotros y para aprender de forma colaborativa», señalar que el 77,5\% manifiesta estar de acuerdo o completamente de acuerdo ante esta afirmación. Un 74,7\% del total de la muestra piensa que incorporar redes sociales como Twitter en la enseñanza universita- ria es fundamental en la actualidad (ítem 7), y un $76,6 \%$ valora positivamente la inclusión de la red social Twitter en las clases de Introducción a la Pedagogía Social (ítem 8).

A continuación, presentamos la tabla de estadísticos básicos de cada uno de los ítems utilizados:

Tabla 3. Estadísticos básicos de los ítems del cuestionario

\begin{tabular}{|c|c|c|c|c|c|c|}
\hline Ítem & $\mathbf{N}$ & Media & Mediana & Moda & Desviación & Varianza \\
\hline $\begin{array}{l}\text { 1. Ya conocía la red social Twitter como usuario/a } \\
\text { antes de usarla en clase de Pedagogía Social }\end{array}$ & 107 & 3,62 & 4 & 4 & ,886 & ,786 \\
\hline $\begin{array}{l}\text { 2. Me ha resultado fácil utilizar Twitter durante el de- } \\
\text { sarrollo de la asignatura }\end{array}$ & 107 & 3,38 & 4 & 4 & ,809 & ,654 \\
\hline $\begin{array}{l}\text { 3. Los protocolos establecidos para establecer las } \\
\text { comunicaciones en la red social Twitter fueron } \\
\text { adecuados }\end{array}$ & 107 & 3,44 & 3 & 4 & ,632 & ,400 \\
\hline $\begin{array}{l}\text { 4tilizar la herramienta Twitter como parte de la } \\
\text { metodología de la asignatura me ha parecido útil }\end{array}$ & 107 & 2,86 & 3 & 3 & ,956 & ,914 \\
\hline $\begin{array}{l}\text { 5. Gracias a esta actividad con Twitter, hemos podi- } \\
\text { do afianzar y ampliar los conocimientos sobre el } \\
\text { contenido de la asignatura }\end{array}$ & 107 & 2,76 & 3 & 3 & ,950 & ,903 \\
\hline $\begin{array}{l}\text { 6. La herramienta Twitter nos ha servido para inte- } \\
\text { raccionar entre nosotros y para aprender de for- } \\
\text { ma colaborativa }\end{array}$ & 107 & 2,96 & 3 & 3 & 1,009 & 1,017 \\
\hline $\begin{array}{l}\text { 7. Pienso que incorporar redes sociales como Twit- } \\
\text { ter en la enseñanza universitaria es fundamental } \\
\text { en la actualidad }\end{array}$ & 107 & 3,03 & 3 & 3 & ,936 & ,877 \\
\hline $\begin{array}{l}\text { 8. Valoro positivamente la inclusión de la red social } \\
\text { Twitter en las clases de Introducción a la Pedago- } \\
\text { gía Social }\end{array}$ & 107 & 3,03 & 3 & 3 & ,936 & ,877 \\
\hline
\end{tabular}

Fuente: Elaboración propia a partir de los datos de la encuesta de satisfacción. 


\section{Discusión y conclusiones}

La inclusión de metodologías innovadoras basadas en las nuevas tecnologías en contextos de Educación Superior es una realidad cada vez más tangible. Este tipo de experiencias pedagógicas suponen un avance hacia la adecuación de la docencia universitaria a los nuevos contextos y realidades sociales de esta época de cambios vertiginosos.

El perfil del estudiante universitario ha cambiado, los patrones de conducta hacia el aprendizaje son diferentes en comparación con épocas pasadas, y la universidad tiene la responsabilidad de adaptarse a esos cambios para ofrecer una educación significativa y de calidad, acorde con las nuevas demandas que la sociedad actual exige.

Los resultados obtenidos en la investigación realizada, ponen de manifiesto el impacto positivo que ha generado la inclusión de Twitter como herramienta didáctica en la asignatura de Introducción a la Pedagogía Social, impartida en el primer curso del Grado en Trabajo Social. En este sentido, los estudiantes que han participado en este proyecto de innovación han demostrado una participación activa en las actividades relacionadas con la difusión de información en la red social digital, así como la implicación en la interacción con sus compañeros de estudios.

Se ha logrado establecer una comunidad de aprendizaje estable, aumentando la cohesión grupal y estimulando la cooperación y el trabajo colaborativo. Los estudiantes han experimentado un desarrollo de su capacidad de síntesis, exposición de argumentos y reflexión crítica, así como mejorado sus competencias digitales.

En líneas generales, la muestra objeto de estudio considera apropiada y beneficiosa la incorporación de Twitter en las actividades académicas de la universidad, ayudándoles a afianzar aprendizajes y a ampliar sus conocimientos. La evaluación del proyecto de innovación ha sido procesual, valorando de forma continua los aprendizajes alcanzados por los estudiantes.
La utilización didáctica de Twitter, así como las competencias que los alumnos han adquirido en este proceso, pueden ser transferibles y contextualizadas en cualquier asignatura, materia o titulación. De igual forma, el dominio de Twitter como instrumento de búsqueda y difusión de información, de comunicación e interacción, supone un aprendizaje que acompañará al alumno incluso fuera del entorno académico, adentrándose en los escenarios profesional y relacional.

De igual forma, consideramos que este tipo de experiencias fomenta una importante implicación del alumnado, incidiendo especialmente en la motivación de los estudiantes por la construcción y difusión de los conocimientos, y en la adquisición de sus propios aprendizajes desde la interacción que establecen entre ellos.

\section{Referencias bibliográficas}

Abella-García, V., Delgado-Benito, V., AusínVillaverde, V., \& Hortigüela-Alcalá, D. (2019). To tweet or not to tweet: Student perceptions of the use of Twitter on an undergraduate degree course. Innovations in Education and Teaching International, 56(4), 402-411. https://doi.org/10.1080/14703297.2018.1444503

Acar, S., Neumayer, M., \& Burnett, C. (2019). Social Media Use and Creativity: Exploring the Influences on Ideational Behavior and Creative Activity. Journal of Creative Behavior, 1-14. https://doi.org/10.1002/jocb.432

Adams, B., Raes, A., Montrieux, H., \& Schellens, T. (2018). Pedagogical tweeting in higher education: Boon or bane? International Journal of Educational Technology in Higher Education, 15(1), 19. https://doi.org/10.14434/josotl.v15i2.12825

Aguirre, J., Chaves, S., \& Burban, K. (2019). Adoption, adaptation, and use of mobiles by university students: A Central American example. Journal of Applied Research in Higher Education, 11(3), 493-505.

https://doi.org/10.1108/JARHE-07-2018-0130

Al-Dheleai, Y.M., Tasir, Z., \& Jumaat, N.F. (2020). Depicting Students' Social Presence on 
Social Networking Site in Course-Related Interaction. SAGE Open, 10(1), 1-8. https://doi.org/10.1177/2158244019899094

Alonso, S., \& Alonso, Ma.M. (2014). Las redes sociales en las universidades españolas. Revista de Comunicación Vivat Academia, 126, 54-62. https://doi.org/10.15(2)78/va.2014.126.54-62

Andreev, V.V., Gorbunov, V.I., Evdokimova, O.K., Nikitina, I.G., \& Trofimova, I.G. (2020). Negative aspects of the use of infocommunication technologies by undergraduate students of engineering academic programs in a regional Russian university. Universal Journal of Educational Research, 8(3), 844-856. https://doi.org/10.13189/ujer.2020.080315

Arango, D., Fernández, J., Rojas, Ó., Gutiérrez, C., Villa, C., \& Grisales, M. (2020). Learning styles in university teachers: Evaluation of the relationship between perception and use of ICT in educational environments. RISTI - Revista Iberica de Sistemas e Tecnologias de Informacao, E29, 406-420. https://bit.ly/3eVJjAH

Bautista, M.A., \& Cipagauta, M.E. (2019). Didactic trends and perceived teachers' training needs in higher education: A case study. International Journal of Cognitive Research in Science, Engineering and Education, 7(3), 71-85. https://doi.org/10.5937/IJCRSEE1903071B

Bardin, L. (2002) Análisis de contenido. 3a edición. Akal.

Bidarian, S., Bidarian, S., \& Davoudi, A. (2011). A Model for application of ICT in the process of teaching and learning. Procedia Social and Behavioral Sciences, 29, 1032-1041. https://doi.org/10.1016/j.sbspro.2011.11.336

Castro, J., \& Chirino, E. (2011). Teachers' opinion survey on the use of ICT tools to support attendance-based teaching. Computers \& Education, 56(3), 911-915.

https://doi.org/10.1016/j.compedu.2010.11.005

Cela-Ranilla, J. M., Esteve-González, V., Esteve-Mon, F., González-Martínez, J., \& Gisbert-Cervera, M. (2017). El docente en la sociedad digital: Una propuesta basada en la pedagogía transformativa y en la tecnología avanzada. Profesorado, Revista de Currículum y Formación del Profesorado, 21(1), 403-422. https://bit.ly/2MCDVGs
Cotán, A., Martínez, V., García-Lázaro, I., GilMediavilla, M., \& Gallardo-López, J.A. (2020). El trabajo colaborativo online como herramienta didáctica en Espacios de Enseñanza Superior (EEES). Percepciones de los estudiantes de los Grados en Educación Infantil y Primaria. Revista d'Innovació Docent Universitària, 12, 82-94. https://doi.org/10.1344/RIDU2020.12.9

Corujo-Vélez, C., Gómez-Del Castillo, M.T., \& MerlaGonzález, A.E. (2020). Construtivist and collaborative methodology mediated by ICT in higher education using webquest. PixelBit, Revista de Medios y Educación, 57, 7-57. https://bit.ly/2Uiorf4

Da Silveira, S. A. (2017). Tudo sobre tod@ s: Redes digitais, privacidade e venda de dados pessoais. Edições Sesc.

De Haro, J.J. (2009). Las redes sociales aplicadas a la práctica docente. Didáctica, Innovación y Multimedia, 13, 1-8. (en línea) http://bit.ly/2S4siKM

Domingo-Coscollola, M., Bosco-Paniagua, A., Carrasco-Segovia, S., \& Sánchez-Valero, J.A. (2020). Fomentando la competencia digital docente en la universidad: Percepción de estudiantes y docentes. Revista de Investigación Educativa, 38(1), 167-182. https://doi.org/10.6018/rie.340551

Dommett, E. (2019). Understanding student use of twitter and online forums in higher education. Education and Information Technologies, 24(1), 325-343. https://doi.org/10.1007/s10639-018-9776-5

Eaton, P.W., \& Pasquini, L.A. (2020). Networked practices in higher education: A netnography of the \#AcAdv chat community. Internet and Higher Education, 45,1-10. https://doi.org/10.1016/j.iheduc.2019.100723 Fernández-Ferrer, M., \& Cano, E. (2019). Experiencias de retroacción para mejorar la evaluación continuada: El uso de Twitter como tecnología emergente. Educar, 55(2), 437-455. https://doi.org/10.5565/rev/educar.872

Freites, M. (2017). Twitter como herramienta de aprendizaje en la educación superior europea y latinoamericana: Avances, retos. ¿Qué está por venir? Eduweb, 11(1), 67-75. http://bit.ly/38XEnbC 
Gairín, J., \& Mercader, C. (2018). Uses and abuses of ICT in adolescents. Revista de Investigacion Educativa, 36(1), 125-140. http://dx.doi.org/10.6018/rie.36.1.284001

Garcias, A.P., Tur, G., Mesquida, A.D., \& Marín, V.I. (2020). Reflexive skills in teacher education: A tweet a week. Sustainability, 12(8), 3161. https://doi.org/10.3390/su12083161

García, T., \& Carmona, J. (2014). Profesor y alumnado conectados en una red pública compartida. Revista de Investigación Educativa, 32(2), 463-478. https://doi.org/10.6018/rie.32.2.171801

García, J. L., \& García, R. (2012). Aprender entre iguales con herramientas web 2.0 y twitter en la universidad. Análisis de un caso. EDUTEC. Revista Electrónica de Tecnología Educativa, 40, 1-14. https://doi.org/10.21556/edutec.2012.40.367

García-Suárez, J., Trigueros-Cervantes, C., \& RiveraGarcía, E. (2015). Twitter como recurso para evaluar el proceso de enseñanza universitaria. RUSC. Universities and Knowledge Society Journal, 12(3), 32-44.

https://doi.org/10.7238/rusc.v12i3.2092

Garrote, D., Jiménez-Fernández, S., \& Serna R.M. (2018). Management of time and use of ICT in university students. Pixel-Bit, Revista de Medios y Educación, 53, 109-121. https://doi.org/10.12795/pixelbit.2018.i53.07

Gavilán, D., Martínez-Navarro, G., \& FernándezLores, S. (2017). University students and informational social networks: Total sceptics, dual moderates or pro-digitals. Comunicar, 25(53), 61-70. https://doi.org/10.3916/C53-2017-06

Gleason, B., \& Manca, S. (2019). Curriculum and instruction: Pedagogical approaches to teaching and learning with Twitter in higher education. On the Horizon, 28(1), 1-8. https://doi.org/10.1108/OTH-03-2019-0014

González-Hernando, C., Valdivieso-León, L., \& Velasco-García, V. (2020). Estudiantes universitarios descubren redes sociales y edublog como medio de aprendizaje. RIED. Revista Iberoamericana de Educación a Distancia, 23(1), 223-239.

https://doi.org/10.5944/ried.23.1.24213
Grosseck, G., \& Holotescu, C. (2008). Can we use twitter for educational activities? The 4th International Scientific Conference eLSE "eLearning and Software for Education", Bucharest, April, 1-11. http://bit.ly/36JE5Ud

Halliwell, J. (2020). Applying Social Media Research Methods in Geography Teaching: Benefits and Emerging Challenges? Journal of Geography, 119(3), 108-113. https://doi.org/10.1080/00221341.2020.1755717

Htay, M., McMonnies, K., Kalua, T., Ferley, D., \& Hassanein, M. (2020). Postgraduate students' perspective on using Twitter as a learning resource in higher education. Journal of Education and Health Promotion, 9(1), 1-7. https://doi.org/10.4103/jehp.jehp_321_18

Hortigüela-Alcalá, D., Sánchez-Santamaría, J., PérezPueyo, Á., \& Abella-García, V. (2019). Social networks to promote motivation and learning in higher education from the students' perspective. Innovations in Education and Teaching International, 56(4), 412-422. https://doi.org/10.1080/14703297.2019.1579665

Jami Pour, M., \& Taheri, F. (2019). Personality traits and knowledge sharing behavior in social media: Mediating role of trust and subjective well-being. On the Horizon, 27(2), 98-117. https://doi.org/10.1108/OTH-03-2019-0012

López-Noguero, F., \& Cobos, D. (2016). Redes sociales y educación para el desarrollo: Espacios para la promoción de una ciudadanía global. En T. Terrón y M. Esteban (Coord.), Ciudadanía mundial y educación para el desarrollo. Una mirada internacional (pp. 1462-1471). Octaedro.

López-Noguero, F., \& Cruz-Díaz, R. (2018). El uso de Twitter en la enseñanza superior como herramienta innovadora en el aula. En L. Torres (Coord.). Innovación docente: Nuevos planteamientos, (pp. 65-73). Octaedro.

Machado, D. (2018). A modulação de comportamento nas plataformas de mídias sociais. En J. Souza., R. Avelino., \& S. Amadeu da Silveira, A sociedade de controle: Manipulação e modulação nas redes digitais (pp.47-69). Hedra.

Malik, A., Heyman-Schrum, C., \& Johri, A. (2019). Use of Twitter across educational settings: A review of the literature. International Journal of Educational Technology in Higher Education, 16(1), 36. 
https://doi.org/10.1186/s41239-019-0166-x

Marín-Díaz, V., \& Cabero-Almenara, J. (2019). Las redes sociales en educación: Desde la innovación a la investigación educativa. RIED. Revista Iberoamericana de Educación a Distancia, 22(2), 25-33. http://dx.doi.org/10.5944/ried.22.2.24248

Martínez, F., \& Acosta, Y. (2011). Integración de las tecnologías de la información y la comunicación (TIC) en la universidad: Diez propuestas de aprendizaje, Revista Mediterránea de comunicación, 2(1), 43-58.

https://doi.org/10.14198/MEDCOM2011.2.03

Martínez-Rodrigo, E., \& Raya-González, P. (2014). El microblogging en el proceso de enseñanzaaprendizaje. Una experiencia académica con Twitter. Historia y Comunicación Social, 18, 139-147.

https://doi.org/10.5209/rev_HICS.2013.v18.44232

Mateo, J. (2004). La investigación ex-post-facto. In R. Bisquerra (Ed.), Metodología de la Investigación Educativa (pp. 195-230). La Muralla

Miller, R.E. (2020). College students and inappropriate social media posting: Is it a question of personality or the influence of friends? Personality and Individual Differences, 158, 109857.

https://doi.org/10.1016/j.paid.2020.109857

Morón, J.A., López-Noguero, F., \& Cobos, D. (2017). El uso de las Redes Sociales en la educación y promoción de la salud. Una experiencia de empoderamiento social en Nicaragua. Profesorado: Revista de currículum y formación del profesorado, 21(4), 439-457. http:// bit.ly/38U1uDQ

Noguera, I. (2015). Rediseño de dos asignaturas incorporando el juego de rol y Twitter para fomentar la motivación de los estudiantes, @ tic. revista d'innovació educativa, 14, 31-40. https://doi.org/10.7203/attic.14.4511

Ozer, M., Sapienza, A., Abeliuk, A., Muric, G., \& Ferrara, E. (2020). Discovering patterns of online popularity from time series. Expert Systems with Applications, 15(2), 113337. https://doi.org/10.1016/j.eswa.2020.113337

O'Keeffe, M. (2019). Academic Twitter and professional learning: Myths and realities. International
Journal for Academic Development, 24(1), 35-46.

https://doi.org/10.1080/1360144X.2018.1520109

Paredes, W., Chaves, L., \& Vera, M. (2020). Communication and Technological Skills to Improve University Teaching Performance. Advances in Intelligent Systems and Computing, 1110 AISC, http://doi.org/385392. 10.1007/978-3-030-37221-7_32

Pérez, A., Cabezudo, S., Morais, A., \& Peañalba, M. (2012) Twitter en la Universidad. Revista del Congreso Internacional de Docencia Universitaria e Innovación, 1, 1-12. http://bit.ly/2u7uIAo

Peña, Ma. A., Rueda, E., \& Pegalajar, Ma. C. (2018). Posibilidades didácticas de las redes sociales en el desarrollo de competencias de educación superior: Percepciones del alumnado. Píxel-Bit. Revista de Medios y Educación, 53, 239-252. https://bit.ly/3h47LBB

Plaza de la Hoz, J. (2018). Benefits and drawbacks of adolescent ICT use: The students' point of view. Revista Complutense de Educacion, 29(2), 491-508. http://dx.doi.org/10.5209/RCED.53428

Prestridge, S. (2014). A focus on students' use of Twitter-their interactions with each other, content and interface. Active Learning in Higher Education, 15(2), 101-115. https://doi.org/10.1177/1469787414527394

Ribeiro, R.M., Bragiola, J.V.B., Eid, L.P., Ribeiro, R.C.H.M., Sequeira, C.A.D.C. \& Pom, D.A (2020). Impact of an intervention through facebook to strengthen self-esteem in nursing students. Revista Latinoamericana de Enfermagem, 28, e3237. https://doi.org/10.1590/15(2)8-8345.3215.3237

Rodríguez, H., \& Restrepo L. F. (2015). Conocimientos y uso del twitter por parte de estudiantes de educación superior. Revista Sophia, 11(1), 44-52. http://bit.ly/38TNZ79

Rojprasert, S., Neanchaleay, J., Boonlue, S., \& Sinlarat, P. (2020). Designing and implementing constructionist learning in a blended advertising photography course. International Journal of Technology Enhanced Learning, 12(1), 20-37. https://doi.org/10.1504/IJTEL.2020.103813

Ruiz, M. \& Fachinetti, V. (2018). Los estudiantes, los sentidos de la formación universitaria. 
InterCambios. Dilemas y transiciones de la Educación Superior, 5(1), 60-69.

https://bit.ly/3cFueSj

Sai-Reddy, V., Kalyan-Reddy, D.S., \& Minu, R.I. (2020). Understating the psychological behavior of twitter post: Through sentiment glossary analysis. International Journal of Psychosocial Rehabilitation, 24(8), 1036-1046. https://bit.ly/30fvn0b

Saeed, N., \& Sinnappan, S. (2011). Adoption of Twitter in higher education, a pilot study. En G. Williams., P. Statham., N. Brown., \& B. Cleland (Eds.), Changing Demands, Changing Directions. Proceedings ascilite Hobart (pp. 1115-1120). University of Tasmania. http://bit.ly/38SW24b

Sánchez-Saus, M., \& Crespo, M. (2018). Social media as a teaching innovation tool for the promotion of interest and motivation in higher education. SIIE 2018 - International Symposium on Computers in Education, 1-5. Jerez. http://doi.org/10.1109/SIIE.2018.8586752.

Siemens, G., \& Weller, M. (2011). Higher education and the promises and perils of social network. Revista de Universidad y Sociedad del Conocimiento (RUSC), 8(1), 164-170. https://doi.org/10.7238/rusc.v8i1.1076

Tess, P.A. (2013). The role of social media in higher education classes (real and virtual). A literature review. Computers in Human Behaviour, 29(5), 60-68.

http://doi.org/10.1016/j.chb.2012.12.032

Thoms, B., \& Eryilmaz, E. (2015). Introducing a twitter discussion board to support learning in online and blended learning environments. Education and Information Technologies, 20(2), 265-283.

http://doi.org/10.1007/s10639-013-9279-3
Universidad Pablo de Olavide (2019). Memoria académica 2018/2019: Resumen estadístico, UPO en Cifras. http://bit.ly/2GJh2hO

Valeria, B., Conny, D.V., \& Patrizi, N. (2018). Cognitive strategies for self-regulation of learning and motivation to study. Construction of average profiles of cognitive functioning and motivational structure for the prevention of drop-out. Journal of Educational, Cultural and Psychological Studies, 17, 139-159. http://dx.doi.org/10.7358/ecps-2018-017-bias

Van der Krogt, F.J. (1998). Learning-network theory: The tension between learning systems and work systems in organizations. Human Resource Development Quarterly, 9(2), 157177.http://doi.org/10.1002/hrdq.3920090207

Van Dijck, J. (2020). Governing digital societies: Private platforms, public values. Computer Law and Security Review, 36, 105377. https://doi.org/10.1016/j.clsr.2019.105377

Van Pujienbroek, T., Poell, R.F., Kroont, B., \& Timmerman, V. (2014). The effect of social media use on work-related learning. Journal of Computer Assisted Learning, 30(2), 159172. https://doi.org/10.1111/jcal.12037

Vázquez-Cano, E., \& Sevillano, M. L. (2019). Uso y funcionalidad didáctica de Twitter desde la perspectiva del estudiante universitario. Un estudio de caso en la UNED. EDUTEC. Revista Electrónica de Tecnología Educativa, 68, 15-29.

https://doi.org/10.21556/edutec.2019.68.1311

Vizcaíno-Laorga, R., Catalina-García, B., \& López de Ayala-López, M.C. (2019). Participación y compromiso de los jóvenes en el entorno digital. Usos de las redes sociales y percepción de sus consecuencias. Revista Latina de Comunicación Social, 74, 554- 572. https://doi.org/10.4185/RLCS-2019-1345 